\title{
PERFIL DE DOMINANCIA CEREBRAL DE ESTUDIANTES DE CIENCIAS ECONÓMICAS Y EMPRESARIALES DE LA UNIVERSIDAD RAFAEL LANDÍVAR
}

Artículo de investigación científica

\section{Guillermo Oswaldo Díaz Castellanos}

Doctor en Sociología, Universidad Pontificia de Salamanca, España; vicedecano de la Facultad de Ciencias Económicas y Empresariales, Universidad Rafael Landívar.

Correo: godiaz@url.edu.gt

Fecha de recepción: 6 de enero de 2020

Fecha de aceptación: 9 de enero de 2020

\section{Resumen}

Este artículo presenta un análisis estadístico descriptivo sobre el perfil de dominancia cerebral o forma de pensamiento de estudiantes de la Facultad de Ciencias Económicas y Empresariales de la Universidad Rafael Landívar, con base en el modelo de Herrmann. También analiza la concordancia entre el perfil de dominancia cerebral y la carrera estudiada, así como la relación entre dicho perfil y el rendimiento académico. Para el efecto, se aplicó la prueba de Herrmann a una muestra de 943 estudiantes de las cinco carreras de la facultad. Los resultados muestran que la mayoría de los estudiantes encuestados tienen un perfil de dominancia cerebral límbico izquierdo, el cual, en general, coincide con la carrera estudiada y que no existe diferencia significativa entre el perfil de dominancia cerebral y el rendimiento académico del estudiante.

Palabras clave: cuadrantes cerebrales, forma de pensamiento, modelo de Herrmann, perfil de dominancia cerebral 


\section{BRAIN DOMINANCE PROFILES IN ECONOMICS AND BUSINESS STUDENTS AT UNIVERSIDAD RAFAEL LANDÍVAR}

Scientific investigation article

\section{Abstract}

This article offers a descriptive statistical analysis of brain dominance profiles in students of the School of Economics and Business at University Rafael Landivar. Based on the Herrmann model, it also analyzes the consistency or alignment between brain dominance profile and career pursued, as well as the relation between said profile and academic performance. To this end, the Herrmann instrument was tested on a sample of 943 students of the five different Economics and Business careers. The results show a limbic left brain dominance profile in the majority of students in the sample group, which in general coincides with the career they pursue. The results did not show meaningful differences between brain dominance profile and academic performance.

Key words: brain quadrants, way of thinking, Herrmann model, brain dominance profile 


\section{Introducción}

El decenio de 1990 fue conocido como la «década del cerebro», debido a que proliferaron investigaciones sobre el funcionamiento del cerebro, entre las que destacó la realizada por Herrmann (1994) por su propuesta de dividir el cerebro en cuatro cuadrantes, que fusionó el modelo de los hemisferios de Sperry (1973) y el de los cerebros límbico y cortical de MacLean (1990).

El modelo de Herrmann se ha utilizado en diversas investigaciones sobre educación, en especial universitaria, entre las que es conveniente mencionar, por su vinculación con este artículo, la de Mendieta y Briones (2017), que identifica el perfil de dominancia cerebral de estudiantes de una universidad de Nicaragua; la de Yarlequé, Navarro, Nuñez, Padilla y Alvarez (2018), que identifica el perfil de dominancia cerebral de estudiantes de primer ingreso en una universidad peruana; y la de Ojeda, Mexicano y Mosqueda (2010), que analiza la evolución de la dominancia cerebral durante un año en estudiantes de una universidad mexicana.

Conocer el estilo de dominancia cerebral es fundamental para la labor educativa universitaria. Salas (2003, p. 167) hace eco de la siguiente pregunta de Sylwester: ¿puede una profesión encargada de desarrollar un cerebro efectivo y eficiente permanecer desinformada respecto al cerebro? El espíritu de la interrogante es motivar el interés de los docentes, en este caso universitarios, por conocer cómo los estudiantes utilizan su cerebro para aprender, ya que como lo mencionan Ojeda et al. (2010) el aprendizaje del estudiante se relaciona con su estilo de pensamiento. En tal sentido, el presente artículo pretende aportar conocimiento sobre cuál es el perfil de dominancia cerebral o forma de pensamiento de estudiantes de la Facultad de Ciencias Económicas y Empresariales de la Universidad Rafael Landívar, así como cotejar si dicho perfil se adecúa a la carrera que estudian. 


\section{Revisión literaria}

En la literatura sobre el tema se encuentran diversos modelos o estilos que teorizan sobre la forma como funciona el cerebro. Entre los más relevantes se pueden mencionar la teoría de los hemisferios izquierdo y derecho de Sperry (1973), por la que ganó el Premio Nobel de Medicina en 1981. Según el citado autor, el hemisferio izquierdo domina el pensamiento lógico, matemático, racional y analítico; mientras que el hemisferio derecho, el pensamiento espontáneo e intuitivo.

La teoría de MacLean (1990) sostiene que el cerebro está formado por tres cerebros en uno (cerebro triuno), que son reptiliano, límbico y neocorteza. El primero se relaciona con lo instintivo, el segundo con los sentimientos y el tercero con la inteligencia.

Con base en el modelo del cerebro triuno, Austin (1997) propuso su modelo de inteligencia múltiples, que son: inteligencia mental, racional, asociativa, intuitiva, emocional, afectiva, estados de ánimo y del comportamiento, básica, de los patrones y de los parámetros. Gardner (1993) también propuso una teoría de inteligencias múltiples, cuyo número estableció en siete: lingüística, lógicomatemática, espacial, musical, corporal y cinética, interpersonal e intrapersonal.

Herrmann (1994), con base en las teorías de Sperry (1973) y MacLean (1990), elaboró su teoría del cerebro compuesto por cuatro cuadrantes. Cada uno representa un diferente estilo de pensamiento que, según el referido autor, es la forma de pensar, crear o aprender. En consecuencia, la dominancia cerebral es la tendencia de las personas a utilizar más las funciones de un hemisferio que del otro en su interacción con la realidad. La siguiente gráfica ilustra el modelo de cuatro cuadrantes de dominancia cerebral propuesto por Herrmann: 


\section{Figura 1. Modelo de los cuadrantes cerebrales de Herrmann}

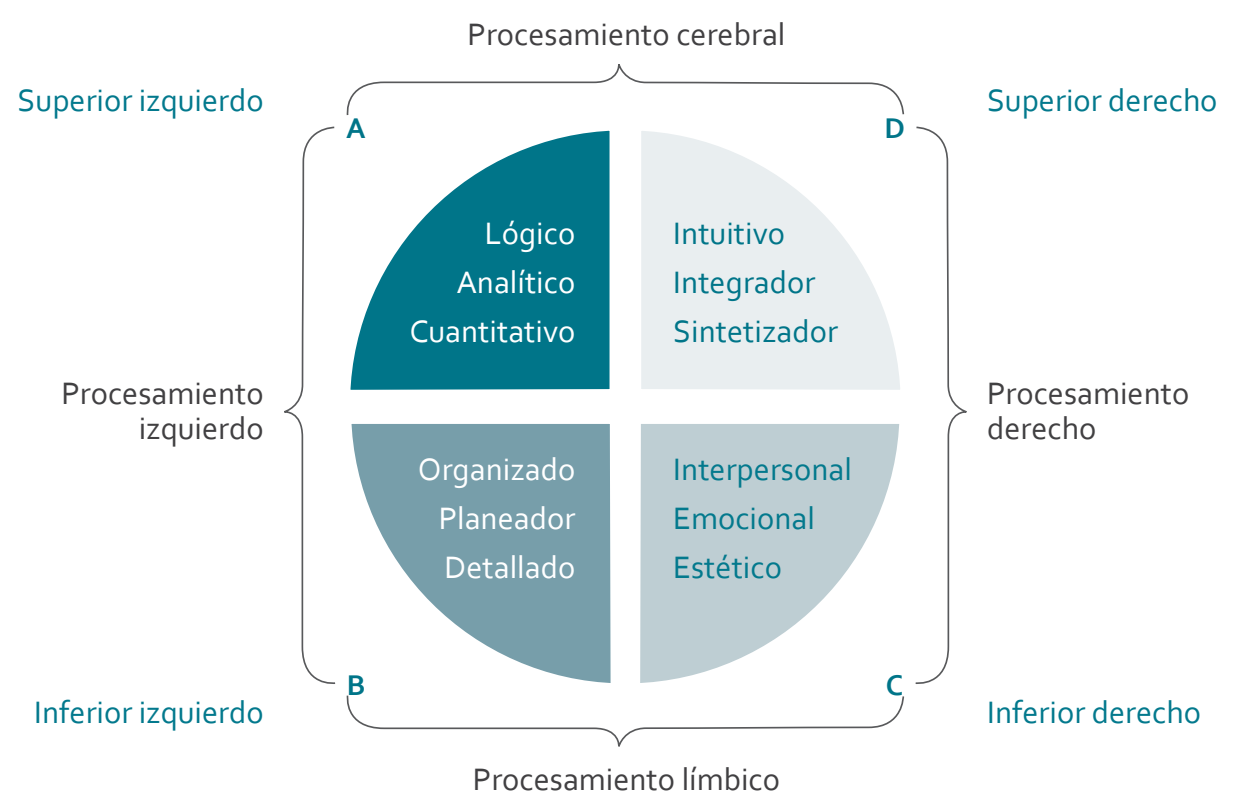

Fuente: Herrmann (1994).

El cuadrante A es llamado «cortical izquierdo» y es de un perfil analítico-experto, con alta inteligencia y capacidad de análisis y razonamiento. El cuadrante B corresponde al «límbico izquierdo» y denota una persona conservadora y organizadora, con capacidades de planificación y administración. El cuadrante $C$ es denominado «límbico derecho» y corresponde a una persona comunicadora, por lo que entre sus capacidades destacan las relaciones interpersonales. El cuadrante $D$ es llamado «cortical derecho» y caracteriza a una persona estratega, con capacidades de innovación y emprendimiento (Velásquez, Remolina y Calle, 2007).

La combinación de la dominancia de los cuadrantes da como resultado los siguientes cuatro tipos de pensamiento, según Velásquez, Remolina y Calle (2006):

5. Realista o de sentido común: conformado por el hemisferio izquierdo, constituido por los cuadrantes A y B.

6. Idealista o kinestésico: corresponde al hemisferio derecho, conformado por los cuadrantes C y D.

7. Pragmático o cerebral: conformado por el área cortical, integrada por los cuadrantes A y D.

8. Instintivo o visceral: correspondiente al sistema límbico, constituido por los cuadrantes B y $C$. 
Existe relación entre el tipo de dominancia cerebral y la elección de la profesión (Chulvi, Felip, García, Galán y Mulet, 2013). Las personas con dominancia cerebral del cuadrante A prefieren elegir profesiones en las áreas de ingeniería y finanzas. Quienes tienen dominancia del cuadrante B se inclinan por profesiones relacionadas con administración y contabilidad. En las personas que predomina el cuadrante C eligen profesiones relacionadas con ciencias sociales, tales como educación y sociología, mientras que las personas con predominio del cuadrante $D$ se orientan a profesiones de contenido artístico y creativo, como lo son marketing, publicidad y diseño gráfico. 


\section{Método}

Para identificar el perfil de dominancia cerebral de los estudiantes de la Facultad de Ciencias Económicas y Empresariales de la Universidad Rafael Landivar, se utilizó la prueba elaborada por Jiménez (2005), con base en los lineamientos teóricos de Herrmann. La prueba está integrada por cuarenta enunciados, diez para cada cuadrante. El participante de la prueba debe calificar en una escala de 1 a 5 cada enunciado, siendo 5 igual a «mejor» y 1 igual a «peor». Las calificaciones otorgadas a los diez enunciados de cada cuadrante se suman y el resultado se multiplica por dos, para obtener el perfil de dominancia cerebral. Una calificación entre 80 y 100 en un cuadrante indica que se tiene dominancia primaria o alta en el mismo. La calificación entre 6 o y 79 revela una dominancia secundaria o regular. Un resultado entre o y 59 evidencia una dominancia terciaria o baja. Por ejemplo, si una persona obtiene 90 en el cuadrante A y 65 en los otros cuadrantes, su perfil de dominancia primaria es el cuadrante A (cortical izquierdo), es decir, lógico-analítico.

La prueba fue aplicada a 943 estudiantes de las licenciaturas de Administración de Empresas (358), Marketing (331), Administración de Hoteles y Restaurantes (74), Contaduría Pública y Auditoría (73) y Economía Empresarial (107), inscritos durante el segundo semestre de 2019. En la aplicación de la prueba se contó con el apoyo de los estudiantes de primer año del curso de Técnicas Básicas de Investigación, a cargo de los profesores Boris Moncada y Estuardo Díaz. 


\section{Resultados}

Los resultados revelan que la mayoría de los estudiantes de la muestra, con $32 \%$, tienen un perfil de dominancia límbico izquierdo, seguido por el perfil cortical izquierdo, con $22 \%$. En total, en el $54 \%$ de los estudiantes de la muestra existe dominancia cerebral izquierda (ver tabla 1). La dominancia cerebral derecha existe en el $30 \%$ de los estudiantes de la muestra y el $16 \%$ restante tiene dominancia múltiple, lo que significa que utiliza indistintamente más de un cuadrante en que se divide el cerebro, según el modelo de Herrmann.

\section{Tabla 1. Perfil de dominancia cerebral}

\begin{tabular}{|l|l|l|}
\hline \multicolumn{1}{|c|}{ Perfil de dominancia cerebral } & \multicolumn{1}{|c|}{$\begin{array}{c}\text { Frecuencia } \\
\text { absoluta }\end{array}$} & $\begin{array}{c}\text { Frecuencia } \\
\text { relativa }\end{array}$ \\
\hline Cortical izquierdo (A) & 207 & $22.0 \%$ \\
\hline Límbico izquierdo (B) & 303 & $32.1 \%$ \\
\hline Límbico derecho (C) & 123 & $13.0 \%$ \\
\hline Cortical derecho (D) & 157 & $16.6 \%$ \\
\hline Múltiple & 153 & $16.2 \%$ \\
\hline Total de la muestra & 943 & $100.0 \%$ \\
\hline
\end{tabular}

Fuente: elaboración propia con base en datos de la encuesta.

Los resultados de este estudio, respecto a la mayoritaria dominancia del cuadrante límbico izquierdo, coinciden con los obtenidos en las investigaciones realizadas por Mendieta y Briones (2017) con estudiantes universitarios de Nicaragua, y la de Yarlequé et al. (2018) con estudiantes universitarios de Perú. Asimismo, coinciden con los resultados de los estudios realizados a estudiantes universitarios de México por Gómez, Recio y Gómez (2010) y de Chile por Herrera, Gazmudi, Pereda y Ramírez (2019).

Los estudiantes de la muestra presentan un mayor uso del lado izquierdo del cerebro $y$, en consecuencia, una menor utilización del lado derecho. Lo anterior revela una baja capacidad creativa, innovadora y estratégica, así como de comunicación y negociación. De ser el perfil de la muestra representativo de los estudiantes de la facultad, la educación debe orientarse a promover más la utilización del lado derecho del cerebro. 
En relación con el tipo de dominancia cerebral, en los resultados de la muestra se observa similitud en lo que respecta a dominancia simple y dominancia doble, ambos con $22 \%$ del total de casos (ver tabla 2). En el primer caso predomina el cuadrante límbico izquierdo y en el segundo, dicho cuadrante en conjunto con el de cortical izquierdo. También se aprecia similitud entre dominancia triple y cuádruple, con $20.1 \%$ y $19.6 \%$, respectivamente. La mayor frecuencia, tanto absoluta como relativa, de la dominancia simple y doble respecto a la triple y cuádruple refuerza lo dicho respecto a la subutilización del cerebro en su totalidad.

\section{Tabla 2. Tipo de dominancia cerebral}

\begin{tabular}{|l|l|l|}
\hline \multicolumn{1}{|c|}{ Tipo de dominancia cerebral } & $\begin{array}{c}\text { Frecuencia } \\
\text { absoluta }\end{array}$ & $\begin{array}{c}\text { Frecuencia } \\
\text { relativa }\end{array}$ \\
\hline Dominancia simple & 209 & $22.2 \%$ \\
\hline Dominancia doble & 213 & $22.6 \%$ \\
\hline Dominancia triple & 190 & $20.1 \%$ \\
\hline Dominancia cuádruple & 185 & $19.6 \%$ \\
\hline Sin dominancia & 146 & $15.5 \%$ \\
\hline Total de la muestra & 943 & $100.0 \%$ \\
\hline
\end{tabular}

Fuente: elaboración propia con base en datos de la encuesta.

Los resultados respecto al tipo de dominancia cerebral también coinciden con los de Mendieta y Briones (2017) y con los de Velásquez, Remolina y Calle (2007), en lo relativo a la mayor incidencia de los tipos de dominancia simple y doble. A su vez, difieren de los obtenidos por Yarlequé et al. (2018), en los que el tipo con mayor frecuencia fue sin dominancia.

La puntuación que permite identificar el perfil de dominancia cerebral según licenciatura cursada por los estudiantes se muestra en la tabla 3. En tres de las cinco carreras se observa una dominancia doble. En las licenciaturas de Administración de Empresas y Contaduría Pública y Auditoría los perfiles dominantes son B o límbico izquierdo, y A o cortical izquierdo. En la licenciatura en Economía Empresarial los dos perfiles dominantes son A y B. En las licenciaturas de Marketing y Administración de Hoteles y Restaurantes se observa una dominancia primaria del perfil B y secundaria del perfil A y D. 
Tabla 3. Dominancia cerebral según licenciatura

\begin{tabular}{|l|c|c|c|c|}
\hline \multicolumn{1}{|c|}{ Licenciatura } & A & B & C & D \\
\hline Administración de Empresas & 80 & 81 & 76 & 77 \\
\hline Administración de Hoteles y Restaurantes & 77 & 80 & 75 & 77 \\
\hline Contaduría Pública y Auditoría & 82 & 84 & 74 & 76 \\
\hline Economía Empresarial & 82 & 81 & 75 & 78 \\
\hline Marketing & 78 & 82 & 76 & 78 \\
\hline
\end{tabular}

Fuente: elaboración propia con base en datos de la encuesta.

Los perfiles de dominancia cerebral corresponden a la licenciatura cursada por los estudiantes, en especial en los casos de Administración de Hoteles y Restaurantes y Contaduría Pública y Auditoría, en los que el perfil dominante es B, el cual como ya se dijo está relacionado con profesiones en las áreas administrativa y contable. En los casos de Administración de Empresas y Economía Empresarial la existencia de dominancia doble, debido a la proximidad del promedio de la prueba en los cuadrantes $A$ y $B$, no permite observar con claridad la relación entre el perfil de dominancia cerebral y la licenciatura estudiada. A efecto de corroborar mejor dicha relación, en la tabla 4 se presenta la frecuencia de dominancia cerebral por licenciatura.

Tabla 4. Frecuencia de dominancia cerebral según licenciatura

\begin{tabular}{|l|c|c|c|c|c|}
\hline \multicolumn{1}{|c|}{ Licenciatura } & A & B & C & D & Total* \\
\hline Administración de Empresas & 84 & 110 & 48 & 63 & 305 \\
\hline Marketing & 57 & 118 & 43 & 57 & 275 \\
\hline Contaduría Pública y Auditoría & 15 & 28 & 6 & 11 & 60 \\
\hline Economía Empresarial & 36 & 21 & 17 & 16 & 90 \\
\hline Administración de Hoteles y Restaurantes & 15 & 29 & 9 & 7 & 60 \\
\hline Total & 207 & 306 & 123 & 154 & 790 \\
\hline *153 no tienen dominancia de un cuadrante & & & & \\
\hline
\end{tabular}

Fuente: elaboración propia con base en datos de la encuesta. 
El total de frecuencias de cada cuadrante según licenciatura evidencia mejor la relación entre el perfil de dominancia cerebral y la licenciatura estudiada. En la licenciatura en Administración de Empresas el mayor número de casos corresponde al perfil B o límbico izquierdo, congruente con lo que postula el modelo del Herrmann. Lo mismo sucede en las licenciaturas en Administración de Hoteles y Restaurantes y Contaduría Pública y Auditoría. También se observa concordancia en la licenciatura en Economía Empresarial, en la que el mayor número de casos es del perfil A o cortical izquierdo, que se caracteriza por un procesamiento lógico, analítico y cuantitativo. En efecto, la licenciatura en Economía Empresarial es la licenciatura con mayor enfoque cuantitativo y analítico. En la licenciatura en Marketing el perfil de dominancia cerebral con mayor número de frecuencias es el $B$, pero según el modelo de Herrmann debiera ser el perfil $D$, que tiene el segundo mayor número de frecuencias, en conjunto con el perfil $A$.

La distribución de frecuencias absolutas de la tabla 4 muestra mayor presencia de estudiantes con dominancia del hemisferio izquierdo del cerebro. Por consiguiente, la mayoría de los estudiantes de la muestra tienen capacidad de resolución de problemas, aunque las soluciones que proponen tienden a ser lógicas y poco creativas. Es la minoría de estudiantes con dominancia del hemisferio derecho del cerebro, en especial del cuadrante D o cortical, los que tienen más desarrolladas las capacidades de creación, innovación, comunicación y expresión, que son fundamentales en el mundo de los negocios del siglo XXI.

En la muestra de estudiantes no se observa una diferencia significativa entre el perfil de dominancia cerebral y el rendimiento académico, medido este a través del promedio de notas. La tabla 5 muestra que el rendimiento académico de los estudiantes es muy similar. El mayor promedio, superior en un punto respecto al promedio de la muestra, corresponde a los estudiantes que tienen dominancia cerebral límbico izquierdo.

\section{Tabla 5. Perfil de dominancia cerebral y rendimiento académico}

\begin{tabular}{|l|l|}
\hline \multicolumn{1}{|c|}{ Perfil de dominancia cerebral } & \multicolumn{1}{|c|}{ Rendimiento académico } \\
\hline Cortical izquierdo (A) & 81.0 \\
\hline Límbico izquierdo (B) & 81.5 \\
\hline Límbico derecho (C) & 79.6 \\
\hline Cortical derecho (D) & 80.2 \\
\hline Múltiple & 80.5 \\
\hline De la muestra & 80.7 \\
\hline
\end{tabular}

Fuente: elaboración propia con base en datos de la encuesta. 
Dado el perfil de dominancia cortical y límbico izquierdo mayoritario existente en la muestra, Sindeev (2018) sugiere, entre otras cosas, utilizar una pedagogía racional centrada en el contenido, con apoyo de hechos y cifras, con la participación de los estudiantes en preparar trabajos orales, en especial que expongan nuevas ideas, para mejor su comunicación. También sugiere enseñarles a globalizar, crear e imaginar. 


\section{Conclusiones}

Los resultados de la prueba de Herrmann muestran que en los estudiantes de la muestra, la dominancia cerebral mayoritaria es la de límbico izquierdo o cuadrante $B$, seguida por la cortical izquierda o cuadrante $A$. En consecuencia, se observa un mayor uso del hemisferio cerebral izquierdo. Lo anterior coincide con resultados de otras investigaciones realizadas con estudiantes universitarios de México, Perú y Nicaragua.

En los estudiantes de la muestra predomina una subutilización del cerebro total, dada la mayor presencia de dominancia simple y doble. Una minoría de estudiantes de la muestra hace un uso global del cerebro. Solo uno de cada cinco tiene dominancia cuádruple.

Existe relación entre el perfil de dominancia cerebral, según el modelo de Herrmann, y la carrera universitaria estudiada, en especial las relacionadas con las áreas de administración y contabilidad. En los estudiantes de marketing la relación es parcial, debido a que la dominancia cerebral cortical derecha es secundaria. 


\section{Referencias}

Austin, E. (1997). Las tres caras de la mente. Caracas: Galac, S. A.

Chulvi, V., Felip, F., García, C., Galán, S. y Mulet, E. (2013). ¿Existe correlación entre los perfiles de los estudiantes y la elección de su especialidad? Ponencia presentada en el 17th International Congress on Project Management and Engineering, Logroño, España.

Gardner, H. (1993). Inteligencias múltiples. La teoría en la práctica. Barcelona: Paidós.

Gómez, A., Recio, R. y Gómez, D. (2010). Diagnóstico de estilos de aprendizaje en estudiantes universitarios de nuevo ingreso basado en la dominancia cerebral. Estilos de Aprendizaje, 5(5), 20-35.

Herrera, E., Gazmuri, M., Pereda, C. y Ramírez, F. (2019). Determinación del perfil de dominancia cerebral en estudiantes de enfermería. Revista Educativa de Ciencias de la Salud, 16(2), 132-136.

Herrmann, N. (1994). The whole brain business. New York: McGraw-Hill.

Jiménez, C. (2005). Inteligencia lúdica. Juego y neuropedagogía en tiempos de transformación. Bogotá: Cooperativa Editorial Magisterio.

MacLean, P. (1990). The triune brain evolution. New York: Plenum Press.

Mendienta, M. y Briones, C. (2017). Dominancia cerebral y educación universitaria. Humanismo y Cambio Social, (10), 102-113.

Ojeda, J., Mexicano, M. y Mosqueda, M. (2011). Evolución de las preferencias de pensamiento en alumnos de la carrera de perfil administrativo, en una institución de educación superior en la Región Laja-Bajío. Pistas Educativas, (96), 88-105.

Salas, S. (2003). ¿La educación necesita realmente de la Neurociencia? Estudios Pedagógicos, (29), 155-171.

Sindeev, A. (2018). Teoría del cerebro total: Plena vigencia para el proceso de enseñanza-aprendizaje actual. Revista de investigación de la Universidad Norbert Wiener, (7), 59-71.

Sperry, R. (1973). Lateral specialization of cerebral function in the surgically separated hemispheres. En B. McGuigan y R. A. Schoonover (Eds.), Psychophysiology of thinking (5-19). New York: Academic Press. 
Velásquez, B., Remolina, N. y Calle, M. (2006). El cerebro: Un mundo de posibilidades para el aprendizaje. Bogotá: Universidad Colegio Mayor de Cundinamarca.

Velásquez, B., Remolina, N. y Calle, M. (2007). Determinación del perfil de dominancia cerebral o formas del pensamiento de los estudiantes de primer semestre del programa de bacteriología y laboratorio clínico. Nova, 5(7), 48-56.

Yarlequé, L., Navarro, L., Nuñez, E., Padilla, M. y Alvarez, G. (2018). Perfil de dominancia cerebral en ingresantes a la universidad de Huancayo. Horizonte de la ciencia, 8(15), 121-132. 